\title{
Coronavirus 2019 (COVID-19) Detection Based on Deep Learning
}

\author{
Toqa Abd Ul-Mohsen Sadoon ${ }^{1}$, Mohammed Hussein Ali ${ }^{2 *}$
}

\author{
Authors affiliations: \\ 1) Electronic and \\ Communication Engineering, \\ Al-Nahrain University, \\ Baghdad, Iraq. \\ toqa.am.wd@gmail.com \\ $\left.2^{*}\right)$ Electronic and \\ Communication Engineering, \\ Al-Nahrain University, \\ Baghdad, Iraq. \\ malhayani@gmail.com
}

\section{Paper History:}

Received: $19^{\text {th }}$ Aug. 2020

Revised: $18^{\text {th }}$ Oct. 2020

Accepted: 21 ${ }^{\text {st }}$ Nov. 2020

\begin{abstract}
Deep learning modeling could provide to detected Corona Virus 2019 (COVID-19) which is a critical task these days to make a treatment decision according to the diagnostic results. On the other hand, advances in the areas of artificial intelligence, machine learning, deep learning, and medical imaging techniques allow demonstrating impressive performance, especially in problems of detection, classification, and segmentation. These innovations enabled physicians to see the human body with high accuracy, which led to an increase in the accuracy of diagnosis and nonsurgical examination of patients. There are many imaging models used to detect COVID-19, but we use computerized tomography (CT) because is commonly used. Moreover, we use for detection a deep learning model based on convolutional neural network (CNN) for COVID-19 detection. The dataset has been used is 544 slice of CT scan which is not sufficient for high accuracy, but we can say that it is acceptable because of the few datasets available in these days. The proposed model achieves validation and test accuracy $84.4 \%$ and $90.09 \%$, respectively. The proposed model has been compared with other models to prove superiority of our model over the other models.
\end{abstract}

Keywords: Medical Image, Image Processing, Image Classification, Deep Learning, Convolutional Neural Network, Coronavirus, COVID-19..

$$
\begin{aligned}
& \text { الكشف عن فيروس كورونا } 2019 \text { (COVID-19) بناءً على التعلم } \\
& \text { تقى عبدالمسن سعدون ، محمد حسين علي }
\end{aligned}
$$

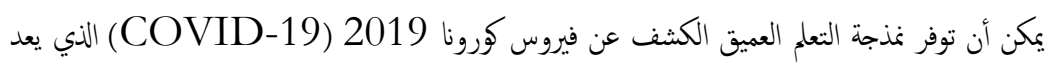

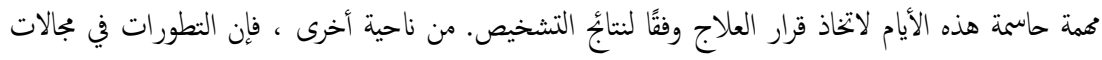

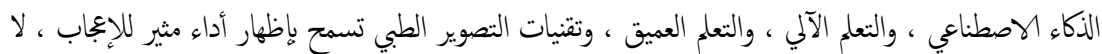

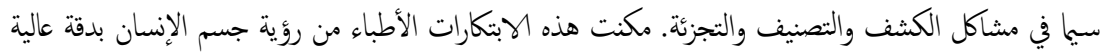

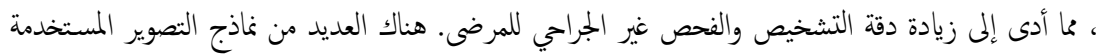

$$
\begin{aligned}
& \text { للكشف عن COVID-19 ، لكننا نستخدم التصوير المقطي المحوسب (CT) لأنه شائع الاستخدام. } \\
& \text { علاوة على ذلك ، نستخدم للكشف عن نموذج التعلم العميق المستند إلى الشبكة العصبية التلافيفية (CNN) }
\end{aligned}
$$

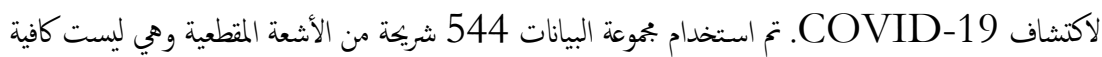

$$
\begin{aligned}
& \text { للحصول على دقة عالية ، ولكن يككنا القول أنها مقبولة بسبب مجموعات البيانات القليلة المتاحة في هذه الأيام. }
\end{aligned}
$$

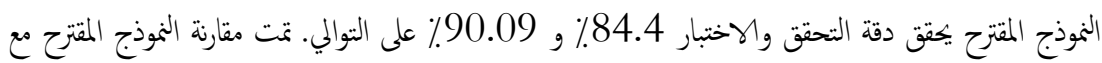

$$
\begin{aligned}
& \text { الناذج الأخرى لإثبات تفوق نموذجنا على الناذج الأخرى. }
\end{aligned}
$$

\section{Introduction}

New coronavirus (COVID-19) formerly known as (2019-nCoV) [1]-[2], has been reported in Wuhan, China [2]-[6], since late December 2019. The personto-person transmission has affected 212 countries around the world $[6,7]$. Overall, COVID-19 is considered an acute disease but can also be fatal, with a $2 \%$ mortality rate. Severe illness may result in death from massive alveolar progressive respiratory damage and failure $[2,8]$. Until this writing moment, there has

NJES is an open access Journal with ISSN 2521-9154 and eISSN 2521-9162

This work is licensed under a Creative Commons Attribution-NonCommercial 4.0 International License 
been a rapid increase in the number of corona disease cases as well as the number of deaths. From the situation report in Word Health Organization-209, 16-August-2020, there are globally 21,294,854 confirmed and 761,779 deaths [9]. Computed tomography (CT) is the preferred imaging method for diagnosing the new COVID19 [10]. However, CT is a well-known medical imaging method used to produce cross-sectional slides (slices) of specific areas of a scanned body. Therefore, the user will be able to see the objects scanned from the inside without cutting. To do this, they use many computerprocessed combinations of X-ray measurements from different angles to photograph the slice [11,12]. Machine learning algorithms such as deep learning play a vital role in the diagnosis of the disease [13]. There are three categories of machine learning, supervised learning, unsupervised learning, and reinforcement learning. In supervised learning, the training data set must consist of the correct input and output together. For example, regression, and classification. While, the training data in unsupervised learning contain inputs only without valid outputs. It is used for clustering. Reinforcement learning uses input, output, and grade in the training data. Such as control and game plays [14]. Deep learning is one types of machine learning algorisms based on used many layers to extract the feature. In contrast, the others machine learning algorithms the feature extraction is independent of the algorithms [15]. Convolutional Neural Network (CNN) or ConvNet, is common deep learning algorithms used for medical image processing, and were largely revived in 2012 when Krizhevky and others proposed a CNN model called Alex-net [16]. Since there is no much research in this felid, we are building a CNN-based deep learning model for classifying a lung CT image to COVID-19 and NonCOVID-19. Then we compare our results with a pre-trained ResNet-50 and Alex-net model. Moreover, we can ignore the accurate classification due to the small input data set. In the future, an accurate model can be built on the available materials, and the accuracy increase about $10 \%$ and reach to $95 \%$ depended on the number of data set.

In [17], Shuai Wang1, et al. proposed a deep learning model to classify CT scan of COVID-19. The model based on extract the Region of Interest from the CT slice and the applied transfer learning neural network based on Inception network. After that, they use Decision tree and Adaboost to improve the classification accuracy. The validation accuracy and testing are $82.9 \%$ and $73.1 \%$ respectively. While Xiaowei Xu1, et al. in [18] used a pre-trained ResNet18 model to classify CT slice of lungs into COVID19, Influenza-A viral pneumonia, and healthy cases. The author firstly candidate the segmentation region as preprocessing step. Then, they use a calculation method to distinguish the structure of the infection as a feature. After that, a pre-trained model was used for classification. The overall accuracy was $86.7 \%$. Currently, the strength of deep learning frameworks and the power of the graphics processing unit (GPU) that helped design, train and validate deep neural networks, in addition to that, helped to develop many models in these years. These models can be run through a high-level programming interface that relies on NVIDIA GPUs accelerated libraries. Common deep learning frameworks are: PyTorch, MXNet, TensorFlow, MATLAB, NVIDA Caffe, Chainer, PaddlePaddle [19]. In this study, we use MATLAB 2018 frameworks, and the powerful of GPU and CUDA from NAVIDA Geforce 920M. This paper is structured in form; in section 2 , the pre-processing and augmentation of data is discussed, then in section 3 the proposed model is discussed with all the details. After that, the experiment and outcome were discussed in Section 4 followed by a conclusion in section 5 .

\section{Data pre-processing and augmentation}

Due to the novelty of the disease, we relied on this data that available in [20] to carry out the research process. These data set contains 349 CT slices of COVID-19, and 195 CT slices of NonCOVID-19. Pre-processing is very important step before the images fed into the models, to correct, adjust, and obtain non-contaminated medical image and this is led to improve the performance, and also decrease the complicity of the models [21]. In this study, we downscale the image into $128 \times 128$ and then applied the Gaussian filter to remove the noise that generate during diagnose from patients moving. Moreover, the data has been shuffled during the training every epoch. The data set is divided into $79 \%$ for training, $20 \%$ for validation, and the remainder for model testing. After that, to reduce the overfitting as well as increase the data set, we augment the data using many methods, such as add salt and paper noise, flipping, rotation with 45, 135, 180 , and 360 angles, and mirror. The data set became 4352 after data augmentation. Fig. 1 and Table 1 show an example of CT slice from the data set and the number of data before and after augmentation.

Table (1): Number of slices

\begin{tabular}{|c|c|c|}
\hline Category & $\begin{array}{c}\text { Number of } \\
\text { slices before } \\
\text { augmentation }\end{array}$ & $\begin{array}{c}\text { Number of } \\
\text { slices after } \\
\text { augmentation }\end{array}$ \\
\hline COVID-19 & 349 & 2792 \\
\hline NonCOVID- 19 & 195 & 1560 \\
\hline Total & 544 & 4352 \\
\hline
\end{tabular}




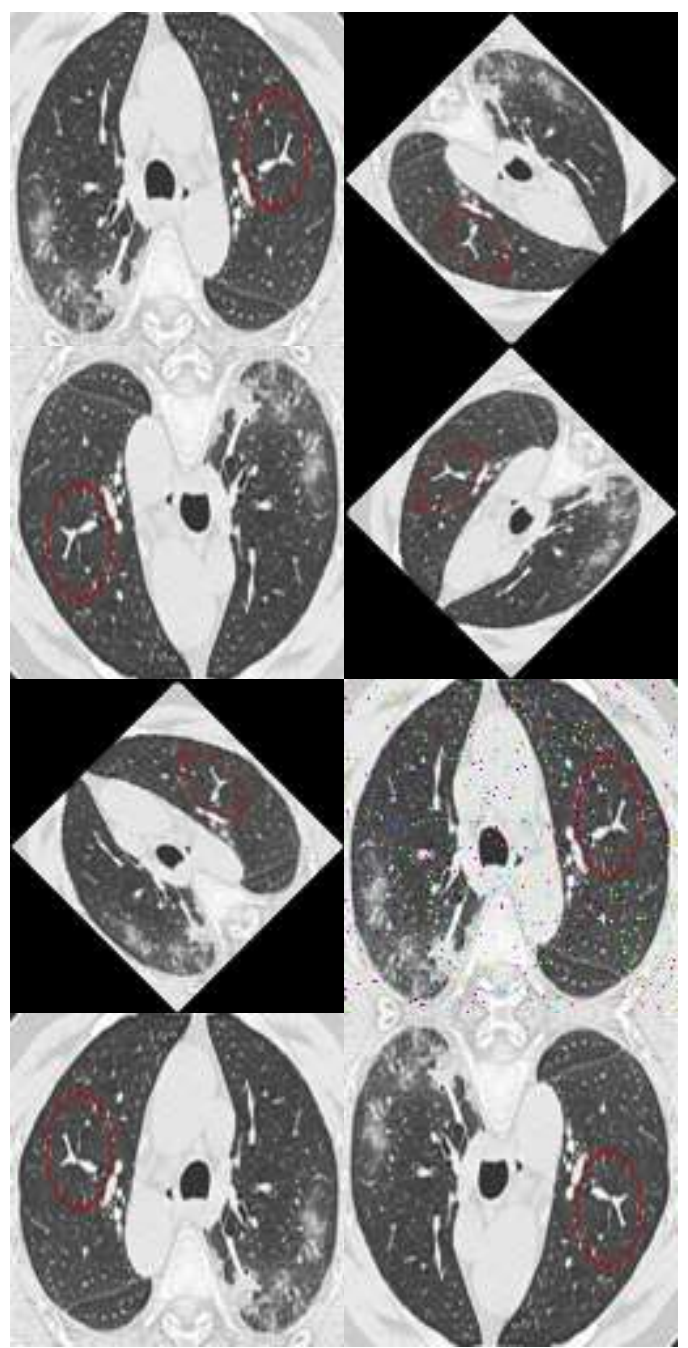

(a)

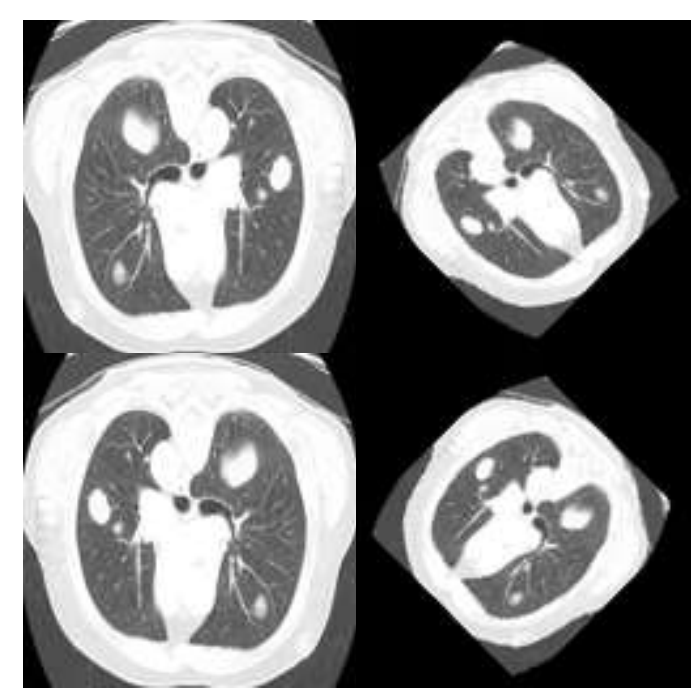

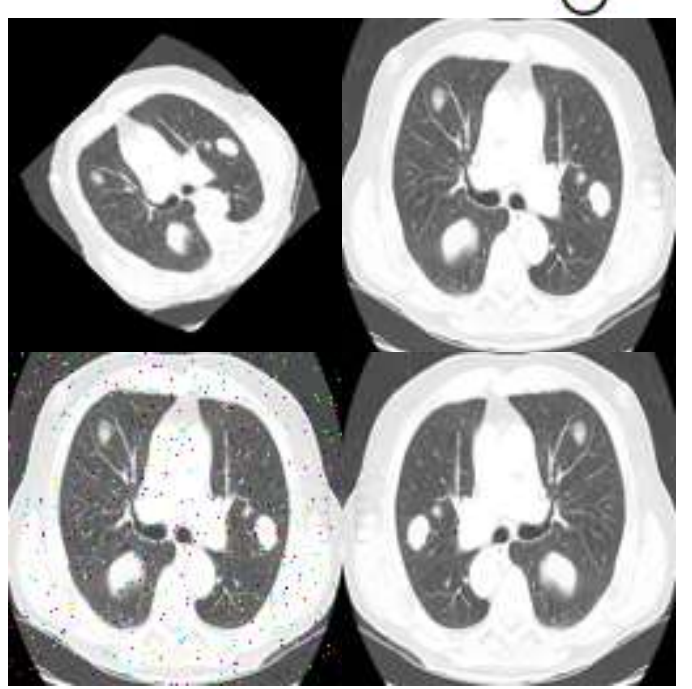

(b)

Figure (1): CT slice after data pre-processing and data augmentation (a) COVID-19, (b) NonCOVID19.

\section{CNN model for covid-19 detection}

$\mathrm{CNN}$ is a deep neural network that has many hidden layers, these layers are used to extract the feature from the input image. The more hidden layers, the more feature extraction [22]. ConvNet include 20 layers began with the input layer that take the input image and at last classification layer which make the last decision for classification, and in between the hidden layers. We should note that, the numbers of layers, and each parameter were selected by trial and error.

\subsection{Convolutional layer}

In this layer, the input image has been convolved with convolutional filters (also called kernel) to extract the feature, and the output is called feature map, which is equal to the number of kernels [13]. In this work, the number of filters is $48,64,96$, and 128 with kernel size $5 \times 5,7 \times 7,9 \times 9$, and $11 \times 11$ respectively. The stride (which is mean by moving the kernel by one step size or more through convolutional operation) size is 1,1,1, and 2. While the padding (which is mean adding more row and column around the image matrix) size is $0,1,2$, and 2 respectively for each convolutional layer. Figure (2) show an example of convolutional layer with kernel size $3 \times 3$, stride size 1 , and padding size 0 .

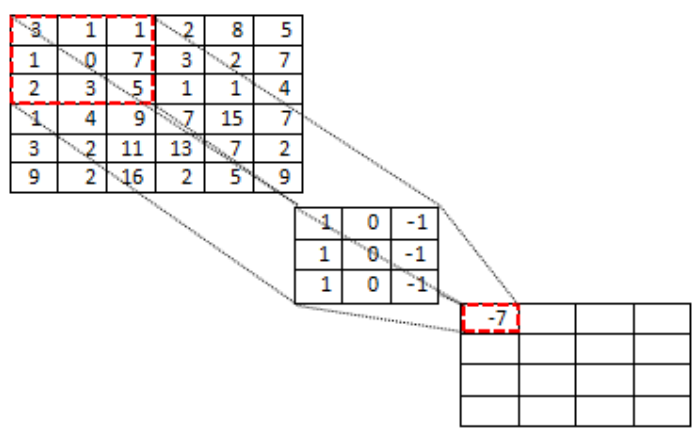

Figure (2): Convolutional layer 


\subsection{Rectifier Linear Unit (ReLU)}

Each convolutional layer followed by ReLU activation function, used to solve vanishing gradient problem through back-propagation algorithm [23]. Figure 3 show the behaviour of ReLU function.

$$
f(x)=\max (0, x)
$$

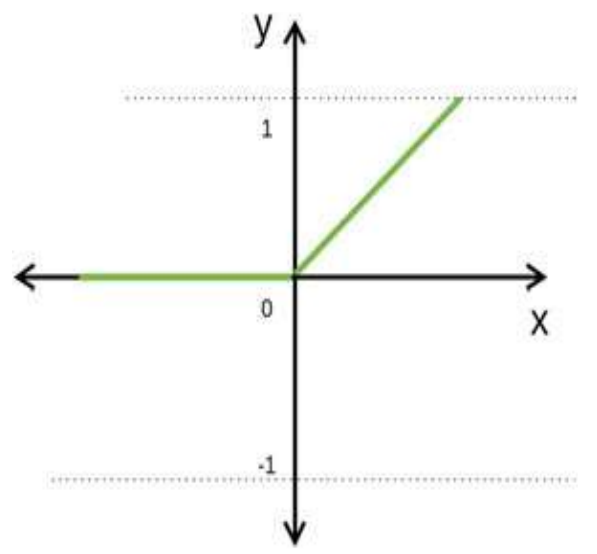

Figure (3): ReLU [24]

\subsection{Pooling layer}

To reduce the dimension of the feature map, and as a result, the complexity of $\mathrm{CNN}$, we can use pooling layer. In this study, we use max pooling layer with size of $2 \times 2$, and stride size of $2 \times 2$ in all $\max$ pooling layers. Figure 4 show the basic work of max pooling layer. Figure (4) show an example of max pooling layer.

\begin{tabular}{|c|c|c|c|c|c|c|}
\hline 10 & 15 & 10 & 17 & & & \\
\hline 20 & 22 & 25 & 32 & $2 \times 2$ max pooling & 22 & 32 \\
\hline 14 & 16 & 30 & 19 & & 21 & 30 \\
\hline 21 & 11 & 13 & 10 & & & \\
\hline
\end{tabular}

Figure (4): Example on max-pooling layer

\subsection{Batch Normalization}

Used to normalize the data set during training processing instead of normalizing all the data set in the pre-processing step, it can be decreasing the training time [25].

\subsection{Dropout Layer}

Dropout layer selected some nodes randomly depended on percentage value. This layer is a common method to prevent overfitting. The dropout ration used in this model was $0.1,0.2$, and 0.3 respectively. Figure 5 show the neural network before and after applied dropout layer.

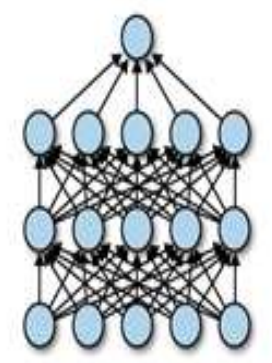

(a) Standard Neurat Net

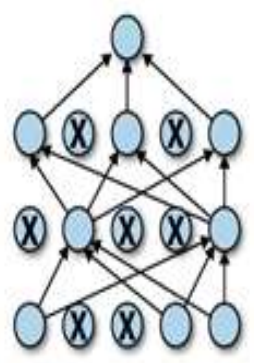

(b)After applying dropout
Figure (5): Dropout layer, (a) Standard Neural Net, (b) After applying dropout [26].

\subsection{Fully-Connected layer}

Finally, the two-dimensional image has been turned into $1 \mathrm{D}$ by using fully connected layer, and connect each neuron to the previous neuron before performs the final result of the classification. In Fig.6, we see the basic neural network represent fully connected layer.

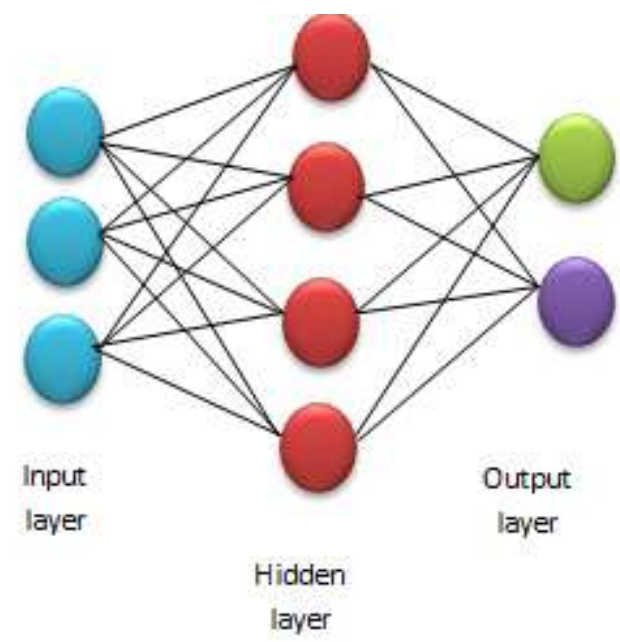

Figure (6): Fully connected layer

\subsection{Softmax Layer}

In this study, softmax layer were used to calculate the probabilities of each categories, and the biggest value of probability represented the correct class. The following equation show the behavior of this function

$$
\mathbf{f}\left(\mathbf{x}_{\mathbf{i}}\right)=\frac{e^{x i}}{\sum_{j=1}^{N} e^{x j}}
$$

Where, $f(x)$ is the activation function output, and $x$ is the input [24].

\subsection{Classification Layer}

In this layer the loss function used to calculate the validation error is cross entropy, which tell us the value of prediction from the actual output, used two parameters; output through learning process and the label in the case of supervised learning algorithms. After that, the weight is update using an optimization method. In our research, we find that the best result obtained when used stochastic gradient descent with momentum (sgdm). Fig. 7 show the block diagram of the proposed model. 


\section{Experiments and result}

In the process of training for deep learning, momentum is set to 0.9 , the initial learning rate is 0.0001 , the maximum iteration 10700 , epoch is 100 , and the mini-batch size is 32 . The training process graph is shown in Fig.8. Looking at the result, we can see that the accuracy of the training shows an increase in curve with respect to the number of iterations. The validation accuracy obtained is $84.4 \%$.
In contrast, there is a decrease in the curve of loss function with respect to the number of iterations, which is about 0.352 . This means that the proposed $\mathrm{CNN}$ structure does well despite the insufficient data set, and there is no overfitting in the training process.

In the case of test step, we use 44 slices for testing and just 4 slices for COVID mislabel as NonCOVID. Figure 9 show some of the predict labels in command window compared with test labels. The testing accuracy is $90.09 \%$.

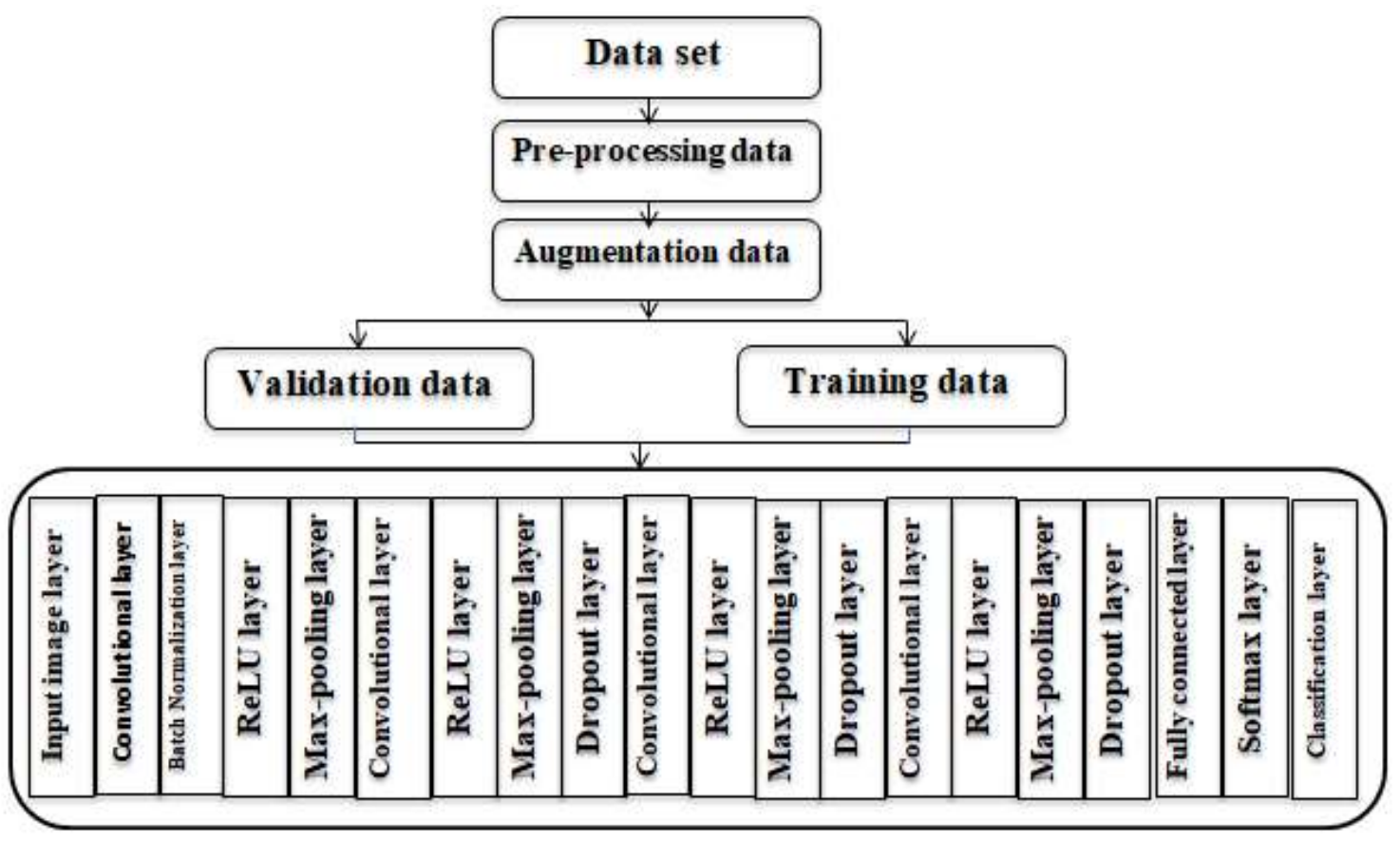

Figure (7): Block diagram of the proposed model

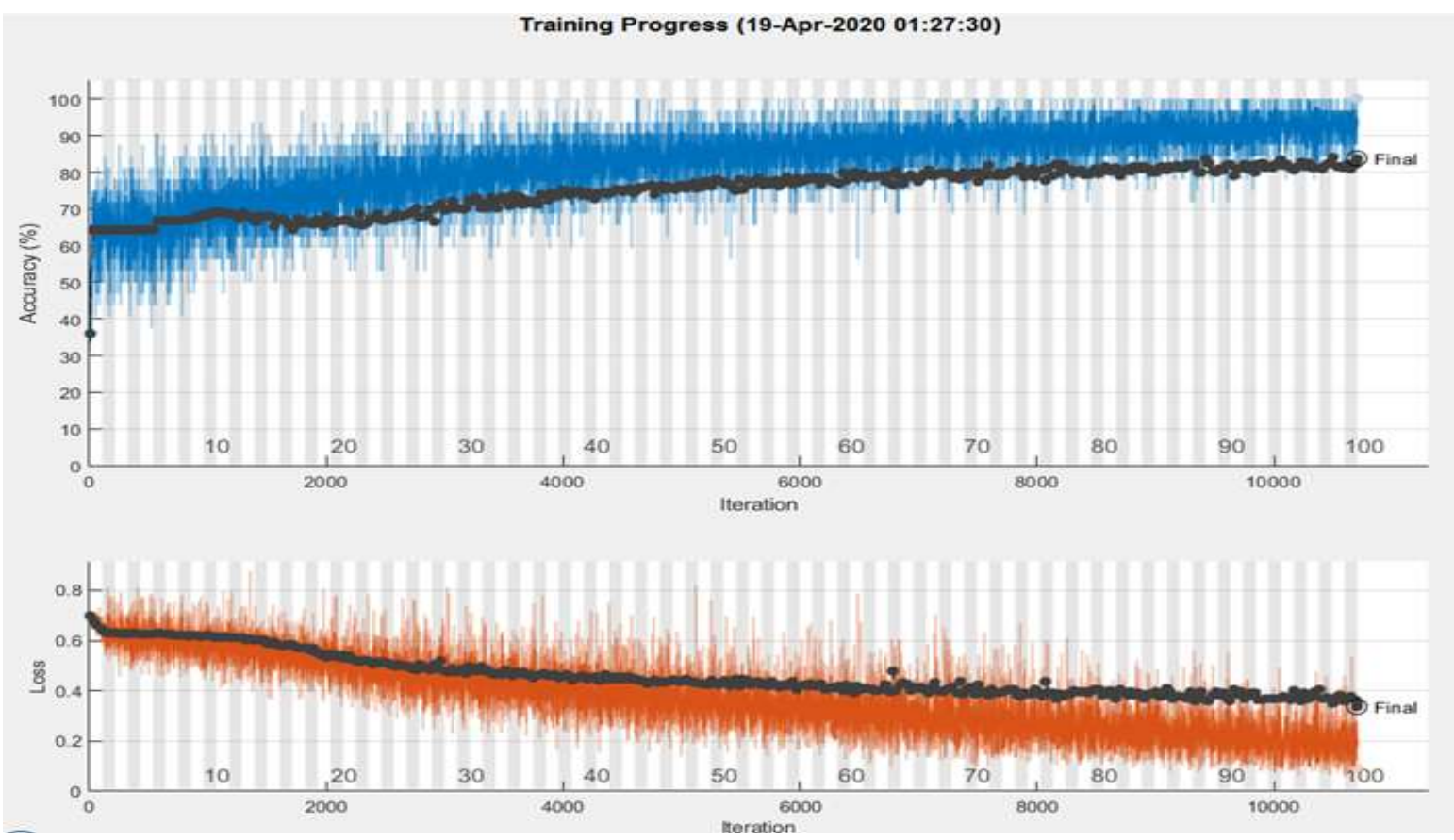

Figure (8) Training progress 


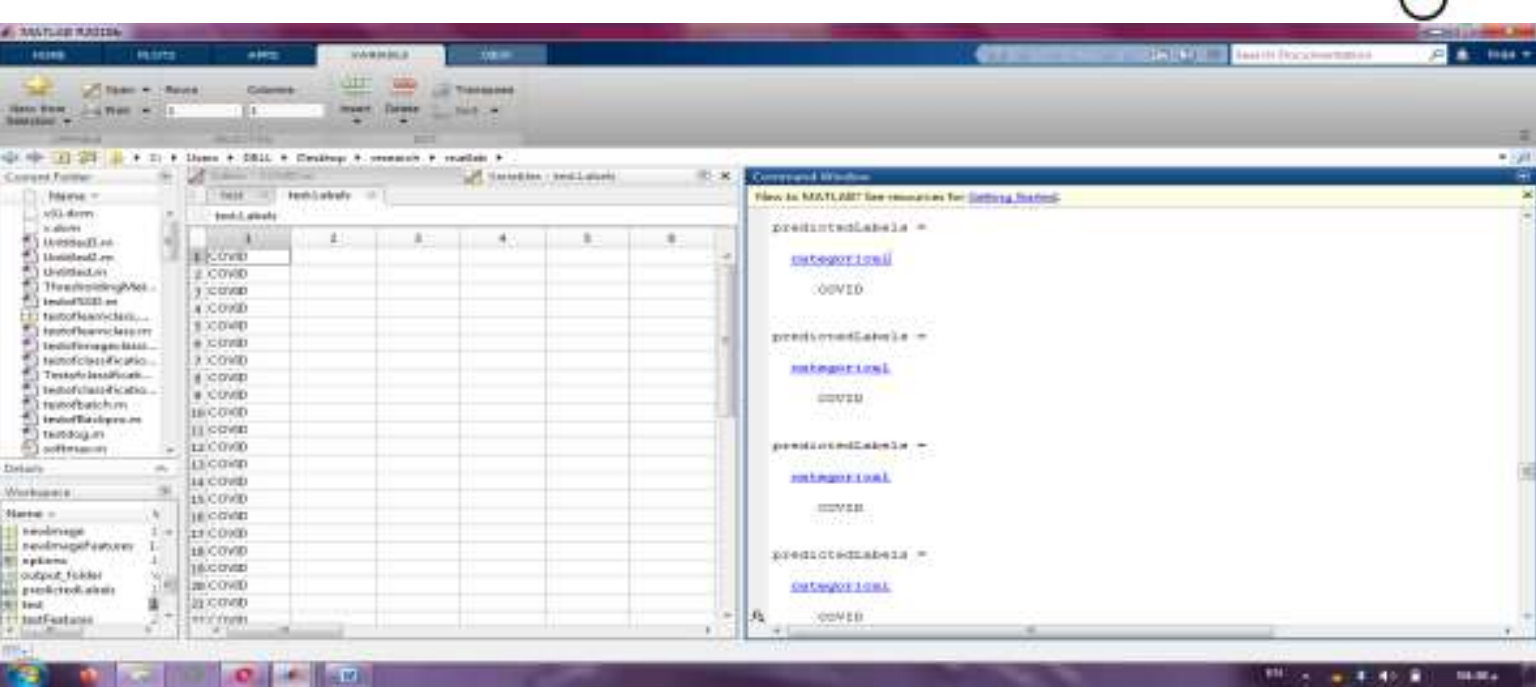

Figure (9) Comparison between actual label and predicted label

\subsection{Number of Layer and Hyper Parameters}

In this subsection, parameters for different structures are included in the training process. Table 2 shows the various previously tested parameters until reaching the structure got a better view performance.

Table 2 Various previously tested parameters and layers.

\begin{tabular}{|c|c|}
\hline Trial and error parameters & Value \\
\hline $\begin{array}{c}\text { Number of (Convolutional } \\
\text { layer+ ReLU+ Pooling } \\
\text { layer) }\end{array}$ & $1,2,3,4,5$ \\
\hline Pooling layer & Max, average pooling \\
\hline Activation function & ReLU, leakyReLU \\
\hline Number of Dropout layer & $1,2,3$ \\
\hline Dropout ratio & $0.1,0.2,0.3,0.4,0.5$ \\
\hline Epoch & $30,70,100$ \\
\hline Learning rate & $0.01,0,001,0.0001$, \\
\hline Optimization methods & SGD, Adam -5 \\
\hline Mini- batch size & 16,32 \\
\hline Number of kernel & $48,64,96,128$ \\
\hline Kernel size & $3,5,7,9,11$ \\
\hline Convolutional layer padding & $0,1,2,3,4$ \\
\hline Convolutional layer stride & 1,2 \\
\hline Pooling layer padding & 0,1 \\
\hline Pooling layer stride & 1,2 \\
\hline
\end{tabular}

\subsection{Time and Tool}

The proposed model for COVID-19 detection is trained on Intel (R) Core(TM) i3-4005U CPU (a)1.7GHz, RAM (4 GB), NVIDIA GeForce 920M GPU, NVIDIA CUDA 10.1.236, and Matlab 2018b . The training time was 253 minutes and $55 \mathrm{sec}$ for training 4,308 images.

\subsection{Confusion Matrix}

The confusion matrices has been used to measure the model's performance for our study. Precision,
Sensitivity, Specificity, and Accuracy have been determined using the following equations:

$$
\begin{aligned}
& \text { Precision }=\frac{\mathrm{TP}}{\mathrm{TP}+\mathrm{FP}} \\
& \text { Sensitivity }=\frac{\mathrm{TP}}{\mathrm{TP}+\mathrm{FN}} \\
& \text { Specificity }=\frac{\mathrm{TN}}{\mathrm{TN}+\mathrm{FP}} \\
& \text { Accuracy }=\frac{\mathrm{TP}+\mathrm{TN}}{\text { total }}
\end{aligned}
$$

Where, TP, FP, TN, FN are true positive, false positive, true negative and false negative, respectively. Looking at the dataset and test model, TP is a positive percentage (COVID-19) that has been correctly classified as COVID-19 by the model, FP is a negative percentage (NonCOVID-19) that is labeled as positive (COVID-19), TN is the negative percentage (NonCOVID-19) that is correctly categorized as NonCOVID-19, and FN is the positive percentage (COVID-19) that is categorized as false (NonCOVID-19) negatively by the model. Table 3 shows the accuracy metrics that are extracted from the confusion matrices show in Fig.10. The precision, sensitivity, specificity and accuracy are $88.5 \%, 87.27 \%, 78.9 \%$, and $84.36 \%$ respectively. Table 4 show compression among our model and the other models. Two pre-trained models ResNet-50, and AlexNet were used in the comparison in addition to [17], and [18]. We should note that, the proposed model has the higher test accuracy in compare with the other models.

Moreover, the simplicity of the proposed model is better than the complexity of the models in $[17,18]$. This gives possibility to obtain better results using the existing data as is without any complications. This type of model can also be applied to other forms of medical images. 


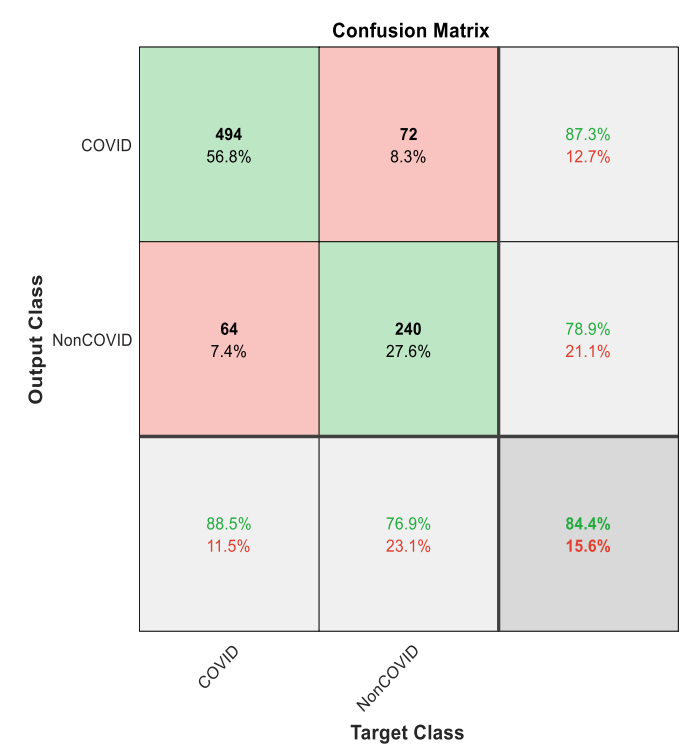

Figure (10): Confusion matrix

Table (3): The accuracy in term of confusion matrix parameters.

\begin{tabular}{|c|c|c|}
\hline Categories & $\begin{array}{c}\text { COVID- } \\
19\end{array}$ & $\begin{array}{c}\text { NonCOVID- } \\
19\end{array}$ \\
\hline TP & 494 & 240 \\
\hline TN & 240 & 494 \\
\hline FP & 64 & 72 \\
\hline FN & 72 & 64 \\
\hline Precision $\%$ & 88.5 & 76.9 \\
\hline Sensitivity $\%$ & 87.27 & 78.9 \\
\hline Specificity $\%$ & 78.9 & 87.27 \\
\hline Accuracy $\%$ & 84.36 & 84.36 \\
\hline
\end{tabular}

Table (4): Comparison between the models

\begin{tabular}{|c|c|c|c|}
\hline Models & $\begin{array}{c}\text { Test } \\
\text { accuracy } \\
\%\end{array}$ & $\begin{array}{c}\text { Validatio } \\
\text { n accuracy } \\
\%\end{array}$ & $\begin{array}{c}\text { Size of the } \\
\text { data set }\end{array}$ \\
\hline $\begin{array}{c}\text { ResNet- } \\
50\end{array}$ & 81.8 & 83 & $\begin{array}{c}544 \text { CT } \\
\text { images }\end{array}$ \\
\hline AlexNet & 84 & 75.5 & $\begin{array}{c}544 \text { CT } \\
\text { images }\end{array}$ \\
\hline $\begin{array}{c}\text { Model in } \\
{[17]}\end{array}$ & 73.1 & 82.9 & $\begin{array}{l}453 \text { CT } \\
\text { images }\end{array}$ \\
\hline $\begin{array}{c}\text { Model in } \\
{[18]}\end{array}$ & - & $\begin{array}{c}\text { Over all } \\
\text { accuracy } \\
(86.7)\end{array}$ & $\begin{array}{l}\text { 618 CT } \\
\text { images }\end{array}$ \\
\hline $\begin{array}{c}\text { Proposed } \\
\text { model }\end{array}$ & $\mathbf{9 0 . 0 9}$ & 84.4 & $\begin{array}{c}544 \text { CT } \\
\text { images }\end{array}$ \\
\hline
\end{tabular}

\section{Conclusion}

In this paper, a $\mathrm{CNN}$ model was established to detect COVID-19 by applying the CNN model to CT slides, and it proved to be a promising complementary diagnostic method for frontline clinicians. Several parameters of $\mathrm{CNN}$ models are used to adapt the system before the last architecture is achieved, and the different architecture and excessive parameters are summarized in Table 2 . The system shows a validation accuracy about $84.4 \%$, and the testing accuracy is $90.09 \%$. However, in this study the proposed system needs to be tested on larger data sets Includes different ages and genders to increase their accuracy. In addition, the classification system architecture cannot be reused in few images because it is one of deep learning determinants.

\section{References}

[1] Wu F, Zhao S, Yu B, et al. "A new coronavirus associated with human respiratory disease in China". Nature 2020; published online Feb 3. DOI:10.1038/s41586-020-2008-3.

[2] Huang C, Wang Y, Li X, et al. Clinical features patients infected with 2019 novel coronavirus in Wuhan, China. Lancet 2020; 395: 497-506 [doi.org/10.1016/S0140-6736(20)30183-5]

[3] Hui D S, Madani T A, Ntoumi F, et al. The continuing COVID-19 epidemic threat of novel coronaviruses to global health-The latest 2019 novel coronavirus outbreak in Wuhan, China. International journal of infectious diseases: IJID: official publication of the International Society for Infectious Diseases 2020;91:264.M. Wegmuller, J. P. von der Weid, P. Oberson, and N. Gisin, "High resolution fiber distributed measurements with coherent OFDR," in Proc. ECOC'00, 2000, paper 11.3.4, p. 109.

[4] Holshue ML, DeBolt C, Lindquist S,et al. First Case of 2019 Novel Coronavirus in the United States. N Engl J Med 2020 Jan 31. doi:10.1056/NEJMoa2001191.

[5] Rothe C, Schunk M, Sothmann P, et al. Transmission of COVID-19 Infection from an 366 Asymptomatic Contact in Germany. N Engl J Med 2020 Jan 30. doi:10.1056/NEJMc2001468.

[6] Paules CI, Marston HD, Fauci AS. Coronavirus infections-more than just the common cold. JAMA. Published online January 23, 2020. doi:10.1001/jama.2020.07571.

[7] Callaway E. Time to use the p-word? Coronavirus enter dangerous new phase. Nature 2020; 579: 12.

[8] Chan JF, Yuan S, Kok KH, et al. A familial cluster of pneumonia associated with the 2019 novel coronavirus indicating person-toperson transmission: a study of a family cluster. Lancet 2020; 395: 514-23.

[9] https://www.who.int/docs/defaultsource/coron aviruse/situation-reports/20200816-covid-19sitrep-209.pdf?sfvrsn $=5$ dde $1 \mathrm{ca} 22$

[10] Guan H, Xiong Y, Shen N, et. Clinical and thin section CT features of patients with COVID-19 pneumonia in Wuhan. Radiologic Practice 2020.

[11] Gabor. T. Herman, "Fundamentals of Computerized Tomography: Image Reconstruction from Projections" second edition Springer 2009.

[12] Marwa. T. Al Hussani and Mohammed H. Ali Al Hayani ,'THE USE OF FILTERED BACKPROJECTION ALGORITHM FOR RECONSTRUCTION OF TOMOGRAPHIC IMAGE" Al-Nahrain University, Collegeof Engineering Journal (NUCEJ) Vol.17 No.2, 2014 pp.151-156.

[13] MI Razzak, S Naz, A Zaib “ Deep Learning for Medical Image Processing: Overview, Challenges and Future "Classification in BioApps, 2018 - 
Springer.[ doi.org/10.1007/978-3-319-659817_12]

[14] Phil Kim, 'MatLab Deep Learning with Machine Learning, Neural Networks and Artificial Intelligence', Seoul (2017).

[15] Yann LeCun, Yoshua Bengio, Geoffrey Hinton, 2015, 'Deep learning', Nuture, vol 521.

[16] Krizhevsky, Alex, "ImageNet Classification with Deep Convolutional Neural Networks," 17 November 2013.

[17] Shuai Wang, et al. "A deep learning algorithm using CT images to screen for Corona Virus Disease (COVID-19)"DOI: 10.1101/2020.02.14.20023028.

[18] Xiaowei Xu1, et al, “ Deep Learning System to Screen Coronavirus Disease 2019 Pneumonia”, 2020 - arxiv.org

[19] https://developer.nvidia.com/deep-learningframeworks

[20] https://drive.google.com/file/d/1odxJF4kyHE tBqhkvz3iXpV3iQK34m6z0/view

[21] 21. R. Ramani,N.Suthanthira Vanitha,S. Valarmathy, 2013, 'The Pre-Processing Techniques for Breast Cancer Detection in Mammography Images', International Journal of Image, Graphics and Signal Processing, vol. 5, no.
5 ,

pp.

$47-$ 54.[ https://doi.org/10.5815/ijigsp.2013.05.06].

[22] Ian Goodfellow, Yoshua Bengio, Aaron Courville, 'Deep Learning', Google books, https://books.google.iq/books?id=Np9SDQAA QBAJ\&printsec $=$ frontcover\&source $=$ gbs ge su mmary $\mathrm{r} \& \mathrm{cad}=0 \# \mathrm{v}=$ onepage\&q\&f$=$ false

[23] Nair, V., Hinton, G.E.: Rectified linear units improve restricted boltzmann machines. In: Proceedings of the 27th International Conference on International Conference on Machine Learning, ICML'10, pp. 807-814. USA (2010).

[24] Ghosh, A., Sufian, A., Sultana, F., Chakrabarti, A., \& De, D. (2020). Fundamental Concepts of Convolutional Neural Network. In Recent Trends and Advances in Artificial Intelligence and Internet of Things (pp. 519-567). Springer, Cham. [https://doi.org/10.1007/978-3-030-32644-9 36]

[25] S. Ioffe and C. Szegedy, "Batch normalization: Accelerating deep network training by reducing internal covariate shift," CoRR, vol. abs/1502.03167, 2015.

[26] Reza Bosagh Zadeh, Bharath Ramsundar, TensorFlow for Deep Learning, https://www.oreilly.com/library/view/tensorflo w-for-deep/9781491980446/ch04.html. 